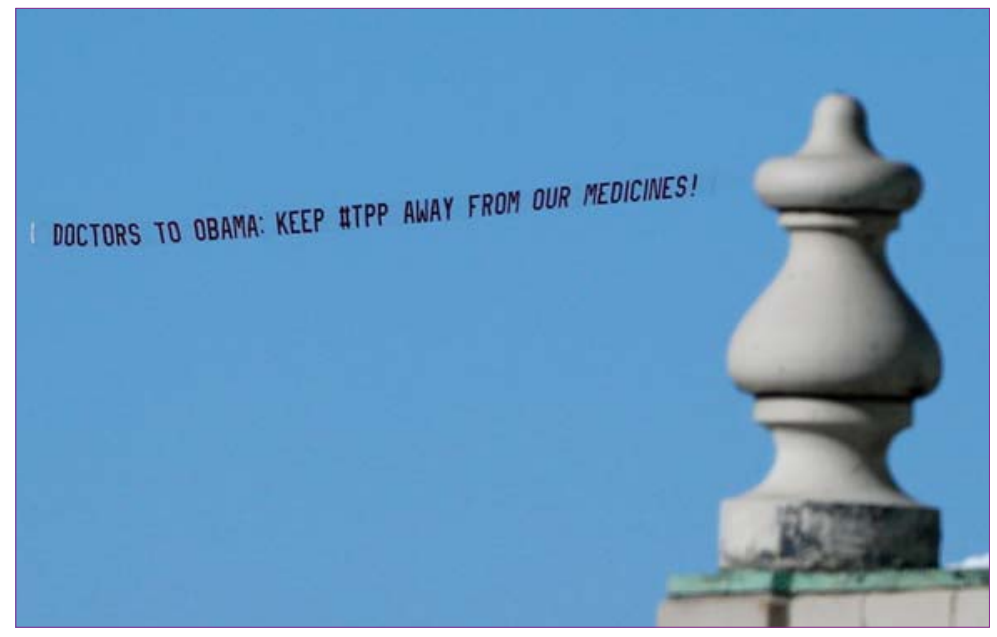

An aerial banner, sponsored by humanitarian organisation Médecins Sans Frontières (MSF), opposing the Trans-Pacific Partnership Agreement with the United States government, is displayed by an aeroplane flying up the Hudson River in New York. MSF claims that intellectual property provisions in the agreement could restrict access to affordable generic medicines, according to its website.

Picture: Shannon Stapleton/Reuters/Picture Media

\section{From the CSIRO}

\section{Manufacturing a human heel in titanium via 3D printing}

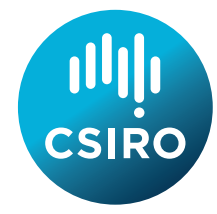

3D printing continues to find new uses as more people get familiar with the capabilities of this relatively new manufacturing tool. Professor Peter Choong at Melbourne's St Vincent's Hospital proved the adage "chance favours the prepared". His patient faced losing a foot due to a cancerous heel, so he came up with the idea of a 3D-printed alternative. He engaged medical device company Anatomics to design the implant. The CSIRO's Lab 22 was contacted due to its capability in 3D printing and, within a week, a foot-saving replacement was used in surgery - a world first.

Using engineering principles and computer-assisted design, Anatomics manipulated the computed tomography data of the patient's healthy heel to design a device to include muscle attachment points to support the new heel once implanted, internal structures to handle the forces of an adult heel, and porosity to reduce implant weight and facilitate tissue integration.

The CSIRO manufactured the design in its Arcam A1 printer. Using titanium Ti6Al4V powder, the printer produced the device overnight. The CSIRO and Anatomics cleaned and prepared the device for surgery. The collaborative team made several prototypes, refining the designs for form, fit and function. The final version was implanted about 24 hours after manufacturing.

There are a number of learning outcomes from this endeavour. The patient was able to keep his foot, which otherwise would have been amputated. Quality of life was preserved. The state of the art has been advanced and, following the success, more challenging applications can be undertaken with lower risk. And from the manufacturing perspective, investment in digital file manipulation, lower-cost materials and capable equipment will make the technology more accessible.

\section{"Precision medicine" star of Obama's State of the Union}

US President Barack Obama's \$215 million Precision Medicine Initiative (PMI) has won the approval of not-for-profit public policy organisation, the Brookings Institute. The PMI's "ultimate goal" is to have scientists build "better tools and databases by using data from nearly one million volunteers", the Brookings Institute reports. "The data will be studied to develop a better understanding of their genetics and personal health traits to in turn, develop individualized treatments." The benefit, the report said, was in "using available science to deliver the most effective treatment possible, while also minimizing the potential for patients to receive unnecessary treatments that do not benefit them".

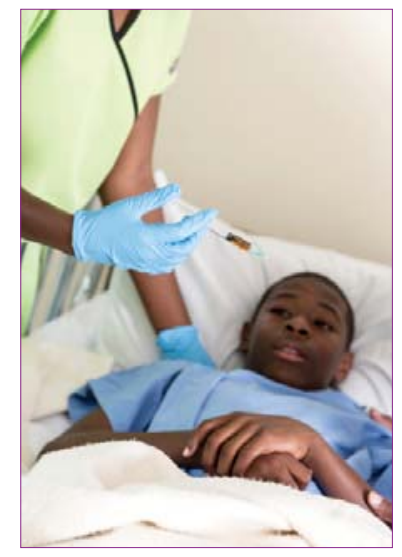

\section{Secret Ebola vaccine trial as WHO re-evaluates epidemic}

As many as 30000 Liberians will be injected with experimental Ebola vaccines over the course of a largescale trial in the West African country, the ABC reports. The "potentially lifesaving" vaccine, which has been delivered to a secret location in Liberia, and initially given to just 12 volunteers, contains a harmless part of the virus scientists hope will prompt the immune system into forming antibodies. Meanwhile, the WHO reports that the focus has now shifted from "slowing transmission to ending the epidemic". "For the first time since the week ending 29 June 2014, there have been fewer than 100 new confirmed cases reported in a week in the 3 mostaffected countries", the latest WHO situation report says. "A combined total of 99 confirmed cases were reported from the 3 countries in the week to 25 January: 30 in Guinea, 4 in Liberia, and 65 in Sierra Leone."

\section{Online monitoring system for sleep apnoea}

Spanish medical technology company Medco has developed an online monitoring system for sleep apnoea patients,

Medical News Today reports. There are estimated to be 1.2 million apnoea patients in Spain, with only $10 \%$ believed to successfully use continuous positive airway pressure (CPAP) treatment. The online system monitors the CPAP devices, relaying information to the Sleep-disordered Breathing Unit of Medco on the use of the device by the patient, as well as on leaks, airflow and other parameters. "Patients will be aware of their ... parameters monthly, helping them to [improve their treatment], beyond pure comfort and relaxation that the use of CPAP provides to the quality of life", the company says.

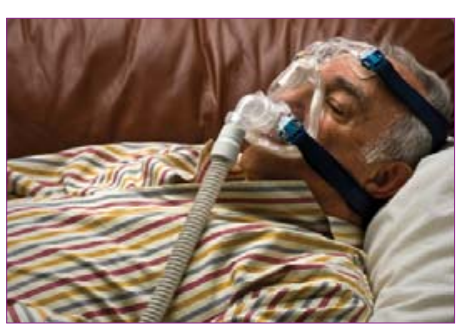




\section{Top 5 MJA articles online}

1) Perspective: What can we do to help Australians die the way they want to?

A different service mix could better meet end-of-life care needs for little additional cost

doi: 10.5694/mja14.01580

2) Clinical focus: Preventing type 2 diabetes: scaling up to create a prevention system

doi: 10.5694/mja14.01153

\section{3) Editorial: What influences doctors to work in}

rural locations?

doi: 10.5694/mja14.01550

4) News from the NHMRC: Can Australia's clinical practice guidelines be trusted?

doi: 10.5694/mjal4.01683

5) Editorial: Childhood food allergy and anaphylaxis: an educational priority

doi: 10.5694/mjal4.01371

\section{Meanwhile, in MJA InSight ...}

1) "Perhaps the findings reinforce the idea ... that patient assessments of quality of care largely reflect bedside manner, surroundings and how they were treated as people, rather than technical expertise - that part of being a good doctor is being a good person."

Mark Ragg: Placebo possibilities

https://www.mja.com.au/insight/2015/3/mark-ragg-placebo-possibilities

2) "Many doctors are aware of drugs with high anticholinergic loads, but what this paper shows [is that] it's the use of multiple medications with lower anticholinergic potency that had the cumulative effect on the total anticholinergic load that the patient was experiencing."

Henry Brodaty: High anticholinergic burden

https://www.mja.com.au/insight/2015/3/high-anticholinergic-burden

3) "Academic research is currently underway looking at complaints handling systems and models of professional regulation in Australia. As the results of this research are not yet known, it is premature to consider any significant changes to complaints and notifications handling in the national scheme."

Georgie Haysom: Complaints concerns

https://www.mja.com.au/insight/2015/3/georgie-haysom-complaints-concerns

\section{Does charitable giving lead to better health?}

The Wall Street Journal reports on a study in the Journal of Economic Psychology which found that "donating to charity may actually improve a giver's physical and emotional well being". "The study also suggested a link between increases in charitable tax subsidies, which have been found to spur giving, and improvements in people's perceptions of their own health." The WSJ quoted the study's author, Associate Professor Baris Yörük from the University at Albany-SUNY, saying: "My findings imply that further expansions in tax subsidies for charitable giving would positively affect the health status of individuals".
MJA Comments The best Comment each month will receive $\$ 50.00$ Email a 100-word comment on any health-related topic to: comments@mja.com.au

Send us your thoughts on this issue's suggested topic:

What do you think about short GP consults? Is the present MBS 'fee' correct?

\section{The chimera of a remote medical workforce}

Why do Australian academics (MJA 19 January 2015) persist in trying to place medical practitioners in remote areas? Where is the evidence that any sizeable country, anywhere in the world, has succeeded?

Tertiary educated professionals have many reasons for choosing not to live away from urban areas.

Many countries have belatedly recognised the value of health care workers in remote areas. Why do we persist in chasing the chimera of a remote medical workforce? It has never worked anywhere. Why do we kid ourselves that Australia is special?

Peter Arnold

Retired General Practitioner, NSW

Congratulations: Owen Lewis (General Practitioner) from SA will receive $\$ 50.00$ for his comment Coordinated screening (MJA 15 Dec 2014, p629).

Visit: www.mja.com.au/journal/mja-instructions-authors-types-articles-published-mja\#Comments

\section{MJA InSight Poll}

Should medical abortions be managed by GPs?

Yes - if they have adequate training

Yes - the evidence is in

No - it needs specialist care

Total respondents: 98

Take part in next week's poll on: www.mja.com.au/insight

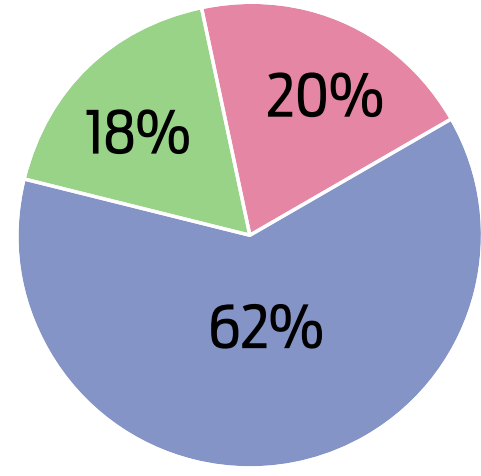

\section{Socialising boosts health literacy}

Research in the Journal of Epidemiology and Community Health reports that older adults who use the internet regularly and take part in social engagement and cultural activities have a better chance of staying informed about health issues as they age. "Support for older adults to maintain socially engaged lives and to access the internet should help promote the maintenance of functional literacy skills during ageing", the authors wrote. Consistent internet use was "protectively associated" with health literacy decline, as was consistent engagement in cultural activities, they found. "As the number of activities engaged in increased, the likelihood of health literacy decline steadily decreased."

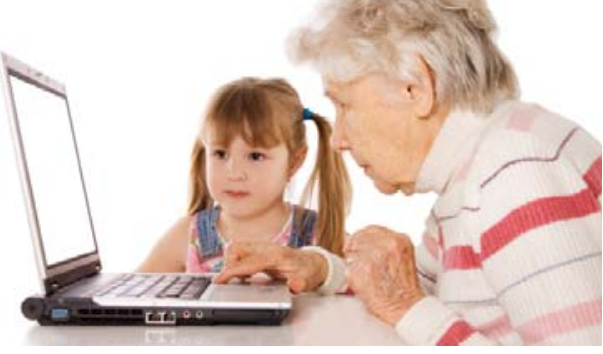

Cate Swannell doi: 10.5694/mja15.n0216 